\title{
The Role of Oxidative Stress in Cocaine Addiction
}

\author{
Tehila Beiser, Rami Yaka* \\ Institute for Drug Research (IDR), School of Pharmacy, Faculty of Medicine, The Hebrew University of Jerusalem, Jerusalem, 91120 , Israel
}

Article Info

\section{Article Notes}

Received: December 27, 2018

Accepted: February 10, 2019

\section{${ }^{*}$ Correspondence:}

Dr. Rami Yaka, Ph.D., The Institute for Drug Research (IDR), School of Pharmacy, The Hebrew University of Jerusalem, Jerusalem, 91120, Israel; Tel: 972-2-6758735; Fax: 972-26758741; Email: yaka@md.huji.ac.il

(c) 2019 Yaka R. This article is distributed under the terms of the Creative Commons Attribution 4.0 International License

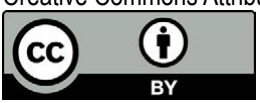

\section{Keywords:}

Cocaine

Oxidative Stress

Addiction

Nitroxides

Tempol

Reactive Oxygen Species
Abstract

Cocaine is a powerfully addictive psychostimulant that elevates dopamine (DA) levels in the mesolimbic system and causes a feeling of wellbeing. At the same time, cocaine leads to toxic effects in many essential organs, including the brain. The harmful effects of cocaine on the brain are the basis for the development of compulsive and irrational behaviors, an integral part of cocaine addiction. Over the last two decades, it has been suggested that the damage and reinforcing properties of cocaine are associated with increased reactive oxygen species (ROS) production. This increase impairs the endogenous defense antioxidant system, either directly by cocaine metabolites, or indirectly via increased DA metabolites, resulting in oxidative stress (OS). It was thus plausible to seek an exogenous, stable and nontoxic antioxidant, which can penetrate the blood brain barrier and counteract the oxidative damage in the brain caused by drugs of abuse such as cocaine. In this minireview we describe studies that explore the role of antioxidants in reducing the OS state in the brain reward system and consequently reversing negative behavioral outcomes induced by cocaine.

\section{Cocaine Addiction}

Cocaine addiction has taken a severe toll not only on drug users themselves but on society as well. The strong craving and impaired judgment associated with cocaine addiction leads to increased and regular drug use despite its harmful consequences. According to the 2018 world drug report, in 2016 there were over 18 million cocaine users worldwide aged 15-64, and the use and demand for cocaine is still growing. Cocaine dependence and craving, representing physical and neuronal damage in the brain, might last years after withdrawal, creating a lifetime risk for relapse $\mathrm{e}^{2}$. Thus, researchers strive to understand the mechanism of cocaine neuronal damage in the hope of finding a suitable treatment.

\section{Cocaine and Oxidative Stress}

Cocaine has been known to be very toxic to many body organs including the liver, the cardiovascular system, and the central nervous system $^{3-5}$. However, the mechanisms for cocaine's toxicity is still poorly understood. Numerous studies suggested that cocaine`s toxicity results in part from oxidative stress $(\mathrm{OS})^{6}$. First, cocaine metabolites, such as norcocaine and its derivatives nitroxide and nitrosonium were found to be even more toxic than cocaine itself in increased reactive oxygen species $(\mathrm{ROS})^{7}$ and in reducing activity of the endogenous protecting antioxidant system, including its main components superoxide dismutase (SOD), glutathione $(\mathrm{GSH})^{8}$ and catalase (CAT) ${ }^{9}$. Second, cocaine blocks re-uptake of dopamine, and as a result elevates dopamine levels. These findings suggest that dopamine and its oxidative metabolites, mainly dopamine quinone, accumulate in the brain and increase ROS production ${ }^{10}$. 
Evidence relating cocaine administration to OS is increasing over the years. One of the first indications for the damage of cocaine to the endogenous antioxidative system was found by Lipton et $\mathrm{al}^{8}$ who reported that even a single prenatal cocaine intake reduces levels of GSH. Another indication was found by Baker et $\mathrm{al}^{11}$ who showed that down-regulation of cystine-glutamate exchanger is involved in cocaine relapse during withdrawal from cocaine self-administration (Box 1). A later study reported that while in the hippocampus of rats the activity of neuronal nitric oxide synthase (nNOS) was increased in response to cocaine, GSH concentration and glutathione peroxidase activity was decreased ${ }^{12}$. Uys et al. ${ }^{13}$ found redox homeostasis changes in glutathione that were associated with OS and plasticity induced behavioral changes in psychomotor sensitization (Box 1) and conditioned reward (Box 1). Taken together, these studies suggest that while ROS are elevated, the glutathione system, as part of the antioxidant defense system, is critically interrupted by acute and chronic exposure to cocaine and may be related to cocaine induced behaviors.

Another aspect of the endogenous antioxidant system is CAT that converts hydrogen peroxide to water. It was reported that the activity of catalase is weakened in response to cocaine in the striatum and pre frontal cortex (PFC) of mice ${ }^{9}$. Since hydrogen peroxide is reported to inhibit dopamine transporters, catalase inactivation results in accumulated toxic effects. On the same line, it was found that the levels of hydrogen peroxide increased in response to a single or repeated cocaine injections, emphasizing the involvement of cocaine in reducing catalase activity ${ }^{14}$. Walker et al. $^{15}$ were the first to evaluate the deficiency of the endogenous antioxidant defense system including CAT, SOD, and GSH in the blood of methamphetamine and cocaine dependent humans. More reports found that exposure to cocaine in utero led to an increase in NO and lipid peroxidation in the hippocampus and cortex ${ }^{16}$ and that cocaine-induced OS provoked cell apoptosis in human neuronal networks ${ }^{17}$. In our previous studies, we found that cocaine induced OS in the nucleus accumbens (NAc) and PFC, two key structures of the reward system, both in slices and following administration of a single or repeated cocaine injections ${ }^{18}$. Similarly, we showed that cocaine induced cell death in PC-12 cell-line through OS $^{19}$. Together, these studies suggest that antioxidants can be suitable candidates to reduce cocaine-induced OS and cocaine-induced behaviors.

\section{Antioxidants Reversed Cocaine-Induced OS and Behaviors}

Tempol (4-hydroxy-2,2,6,6 tetramethylpiperidine-Noxyl), is a stable nitroxide radical ${ }^{20,21}$ that mimics SOD in a catalytic mode and interacts with radicals to prevent radical chain reactions ${ }^{21}$. Tempol has more advantages that make it a good candidate to protect against cocaine-induced OS and subsequent neuronal damage; it easily permeates cell membranes ${ }^{22,23}$, it penetrates and protects the blood brain barrier (BBB) ${ }^{24,25}$ and more importantly, it is a stable radical $^{20,21}$. The biological activity of Tempol in treating OS damage has been studied in diverse areas: it was found to be effective in protecting from radiation ${ }^{26}$ and traumatic head injury ${ }^{27}$; it was reported to prevent taxol-induced neuronal death ${ }^{28}$ and cisplatin-induced ototoxicity in vitro $^{29}$; it prevented cognition impairments in a simulation to Alzheimer's disease ${ }^{30}$, inflammatory pain ${ }^{31}$, short and long-term PTSD-induced memory impairments ${ }^{32}$ and $\beta$-cell death ${ }^{33}$ just to name a few; in addition to other reports on medical applications of nitroxide radicals ${ }^{34,35}$. Here we review only studies presenting the antioxidant effects of Tempol against cocaine-induced OS.

\section{Box 1: Animal Models of Drug-Induced Neuroadaptations and Reward}

Below we describe three of the common animal models that are currently used by addiction researchers to study the neuroadaptations and synaptic plasticity caused by drugs of abuse and the rewarding or positive reinforcing effects of drugs.

\section{Psychomotor Sensitization}

Repeated intermittent treatment with psychomotor stimulants sensitizes animals to the locomotor activating effects of these drugs. Initiation/development phase - animals are systemically injected with the same dose of the drug repeatedly for several days (typically 5 days) and their locomotion activity is monitored continuously. Expression phase - following several weeks (typically 1-3), animals are injected with the drug and locomotor activity is measured.

\section{Conditioned Place Preference (CPP)}

CPP is a Pavlovian (classical) conditioning model. During the training phase one distinct context is paired with drug injections and another context is paired with vehicle injections. During the subsequent testing phase (which is drug-free), the animal's preference for either context is determined by allowing the animal to move between the two contexts. An increased preference for the drug-associated context serves as a measure of the drug's Pavlovian reinforcing (or rewarding) effects.

\section{Drug Self-Administration (SA)}

In this model, animals typically activate a lever via foot press or nose poke to receive contingent drug injections. The premise of this procedure is that drugs of abuse control behavior by functioning as operant positive reinforcers. 
The first evidence for the neuroprotective activity of Tempol against cocaine-induced oxidative stress and the attenuation of cocaine-induced behaviors were obtained in our lab. We found that in sympathetic-like PC12 cells, Tempol significantly reduced cocaine-induced cell death ${ }^{19}$. In addition, we found that two other members of the nitroxide family, Tempo and Tempamine, exhibited a similar effect. Moreover, we confirmed that cocaine induced cell death through the production of ROS by measuring mitochondrial superoxide radical formation and peroxide levels. Indeed, both ROS markers were elevated following cocaine exposure, and decreased when treated with Tempol, indicating that Tempol is able to reverse cocaine-induced cell death. Furthermore, we examined the effect of Tempol in attenuating cocaine-induced $O S$ in animals. We found that Tempol decreased lipid peroxidation and the levels of NO radicals in both the NAc and PFC of rats following a single cocaine injection ${ }^{18}$. Pretreatment with Tempol in PFC and NAc containing brain slices decreased malondialdehyde (MDA); a lipid peroxidation marker; and nitrites levels and increased total antioxidant capacity (TAC), indicating that Tempol may contribute to the endogenous antioxidant defense system. We next evaluated the effect of Tempol on cocaine-induced behaviors. Characteristically, locomotor activity increased in response to repeated injections of the same dose of cocaine and other drugs of abuse. Psychomotor sensitization (PS) reflects the enduring rise in neuronal processes that lie behind addictive behaviors and is also known to be correlated indirectly with drug seeking (see Box 1). We found that in animals treated with Tempol prior to cocaine within the PS paradigm, acute locomotor activity was evident but the development and expression (locomotion activity measured following challenge cocaine injection) of PS was completely abolished. Moreover, we found that in Tempol treated animals, OS was returned to base line levels following the behavioral tests suggesting that Tempol attenuated cocaine PS via the reduction in oxidative stress ${ }^{18}$. Recently, we studied the effect of Tempol on cocaine conditioned reward using the conditioned place preference (CPP) paradigm (Box 1). We demonstrated that injection of Tempol together with cocaine during acquisition of CPP attenuated the expression of CPP measured one day following conditioning ${ }^{36}$. We found that OS was significantly elevated following the establishment of CPP, and that cocaine-induced OS was significantly diminished by pretreatment with Tempol during conditioning. Furthermore, we found that repeated, but not a single, administration of Tempol for seven days during withdrawal from CPP resulted in a significant attenuation in the expression of CPP, without affecting the expression of food reward ${ }^{36}$. Together these results suggest that antioxidants such as Tempol, when administered before or during withdrawal, can interfere with reward processing of drugs of abuse without affecting the response to natural rewards.

Jang et $\mathrm{al}^{37}$ studied the effects of Tempol on cocaine self-administration (SA), a behavioral paradigm that model drug seeking and craving (Box 1). Injecting Tempol before or after the initiation of SA sessions resulted in a significantly reduced number of the active lever presses. These results support the hypothesis that ROS has a critical role in cocaine craving. They further found

Table 1. Evidence for cocaine-induced oxidative stress.

\begin{tabular}{|c|c|c|c|}
\hline Brain area & Cocaine effect on oxidative stress & Paradigm & Reference \\
\hline Fetus brain & $\begin{array}{l}\text { Single cocaine injection decreased endogenous anti- } \\
\text { oxidant glutathione and exogenous antioxidant alpha } \\
\text { Tocopherol but increased their oxidized form }\end{array}$ & $\begin{array}{l}\text { Prenatal effect of single cocaine injec- } \\
\text { tion }\end{array}$ & 8 \\
\hline NAc, PFC and striatum & $\begin{array}{l}\text { Down-regulation of cystine-glutamate exchanger in } \\
\text { cocaine relapse }\end{array}$ & $\begin{array}{l}\text { Withdrawal from self- administration } \\
\text { in rats }\end{array}$ & 11 \\
\hline $\begin{array}{l}\text { Hippocampus and frontal } \\
\text { cortex }\end{array}$ & $\begin{array}{l}\text { Increased nNOS, GSH concentration and glutathione } \\
\text { peroxidase activity is decreased. Memory retrieval is } \\
\text { impaired }\end{array}$ & Morris water maze in rats & 12 \\
\hline NAC & $\begin{array}{l}\text { OS and plasticity is affected by redox homeostasis } \\
\text { changes in glutathione following acute cocaine }\end{array}$ & $\begin{array}{l}\text { Psychomotor sensitization, conditioned } \\
\text { place preference in rats }\end{array}$ & 13 \\
\hline Striatum and PFC & Decreased catalase activity & Single injection of cocaine in mice & 9 \\
\hline Striatum and frontal cortex & $\begin{array}{l}\text { Cocaine increased hydrogen peroxide and induced } \\
\text { glutathione peroxidase and SOD activity }\end{array}$ & $\begin{array}{l}\text { Single and repeated injections of co- } \\
\text { caine in rats }\end{array}$ & 14 \\
\hline Blood & $\begin{array}{l}\text { CAT, SOD, and GSH deficiency and reduction of total } \\
\text { antioxidant capacity }\end{array}$ & $\begin{array}{l}\text { Human blood analysis in cocaine and } \\
\text { methamphetamine dependent users }\end{array}$ & 15 \\
\hline Hippocampus and cortex & increase in NO and lipid peroxidation & In utero in mice & 16 \\
\hline NAc and PFC & Increased lipid peroxidation and nitrites & $\begin{array}{l}\text { Acute and repeated cocaine injections } \\
\text { in rat }\end{array}$ & 18 \\
\hline $\begin{array}{l}\text { Human neuronal progenitor } \\
\text { cells }\end{array}$ & OS leads to cell apoptosis & Acute cocaine treatment & 17 \\
\hline Neuronal-like PC12 cells & Increased OS and cell death & Acute cocaine treatment & 19 \\
\hline
\end{tabular}


that OS occurs mainly in neurons and not in astrocytes, microglia or oligodendrocytes in the NAc of the cocaine SA animals. Moreover, in order to study the involvement of ROS on cocaine reinforcement specifically in the NAc, they microinjected Tempol bilaterally into the NAc prior to cocaine SA and found that the active lever presses were significantly reduced in the Tempol injected group, compared to the saline-injected control group. Systemic injections of Tempol before food SA demonstrated that Tempol did not have any effect on food reinforcement, suggesting that Tempol suppresses cocaine SA without affecting food SA or inactive lever presses, similar to our finding using CPP paradigm ${ }^{36}$.

\section{Drugs of Abuse other than Cocaine}

The role of Tempol in attenuating drug reinforcement was studied using other drugs of abuse. Qi et al ${ }^{38}$ explored the involvement of mGluR5 in morphine CPP and found that microinfusion of MTEP (mGluR5 antagonist) into the NAc shell (the outer substructure of the NAc), attenuated morphine CPP, and blocked the increase of membrane mGluR5 that was observed following morphine CPP in rats. They found that microinjection of Tempol into the NAc shell prevented morphine CPP expression in a similar way to MTEP, but the evidence that mGluR5 and ROS signaling is involved in retrieval of morphine contextual memory was found when microinjection of $\mathrm{t}-\mathrm{BOOH}$, a ROS donor, reversed the effect of mGluR5 antagonist MTEP in attenuating morphine CPP. Jang et $\mathrm{al}^{39}$ found that methamphetamine (METH) induced dopamine release and consequently ROS formation in the NAc. METH-induced ROS were found only in neurons but not in glia, astrocytes or oligodendrocytes. They further showed that systemic administration of Tempol decreased not only dopamine release in the NAc, but it also attenuated METH-induced locomotor activity and the reinforcing effects of METH self-administration in rats, without affecting food intake, similar to what was found with cocaine $e^{36,37}$.

\section{Summary / Conclusions}

In this article, we reviewed the studies that demonstrated the harmful properties of cocaine at the molecular, cellular and behavioral levels which is evidently promoted by ROS formation and become persistent by ROS-induced signaling cascades. In the reviewed studies it is suggested that the harmful consequences of cocaine use could be reversed by employing the antioxidant Tempol as pretreatment and, in some cases, as a treatment following exposure to cocaine. These studies demonstrate a variety of behavioral models: psychomotor sensitization, CPP and self-administration, which together represent a different aspect of addictive behaviors. Thus, these studies suggest that Tempol could be a potential pharmacological treatment not only for cocaine addiction but also to other drugs of abuse.

\section{Acknowledgment}

Supported by the Israel Anti-Drug Authority (IADA) and by the Israel Science Foundation (ISF). R. Yaka is affiliated with the David R. Bloom Center for Pharmacy and the Brettler Center for Research in Molecular Pharmacology and Therapeutics, School of Pharmacy, The Hebrew University of Jerusalem.

\section{References}

1. United Nations Office on Drugs and Crime, U. N. publication. World Drug Report 2018.

2. Hyman SE, Malenka RC. Addiction and the brain : The neurobiology of compulsion and its persistence. Nat Rev Neurosci. 2001; 10: 695-703.

3. Stankowski RV, Kloner RA, Rezkalla SH. Cardiovascular consequences of cocaine use. Trends Cardiovasc Med. 2014; 25: 517-26.

4. Riezzo I, Fiore C, De Carlo D, et al. Side effects of cocaine abuse: multiorgan toxicity and pathological consequences. Curr Med Chem. 2012; 19: 5624-46.

5. Kousik SM, Napier TC, Carvey PM. The effects of psychostimulant drugs on blood brain barrier function and neuroinflammation. Front Pharmacol. 2012 Jun 29; 3: 121.

6. Cunha-Oliveira T, Rego a C Oliveira CR. Oxidative Stress and Drugs of Abuse : An Update. Mini Rev Org Chem. 2013; 10: 321-334.

7. Kovacic P. Role of oxidative metabolites of cocaine in toxicity and addiction: Oxidative stress and electron transfer. Med Hypotheses. 2005; 64: 350-356.

8. Lipton JW, Gyawali S, Borys ED, et al. Prenatal cocaine administration increases glutathione and alpha-tocopherol oxidation in fetal rat brain. Dev Brain Res. 2003; 147: 77-84

9. Macêdo DS, de Vasconcelos SM, dos Santos RS, et al. Cocaine alters catalase activity in prefrontal cortex and striatum of mice. Neurosci Lett. 2005 ; 387: 53-56

10. Smythies J, Galzigna L. The oxidative metabolism of catecholamines in the brain: A review. Biochim Biophys Acta - Gen Subj. 1998;1380: 159-162.

11. Baker DA, McFarland K, Lake RW, et al. Neuroadaptations in cystineglutamate exchange underlie cocaine relapse. Nat Neurosci. 2003; 6: 743-749.

12. Muriach M, López-Pedrajas R, Barcia JM, et al. Cocaine causes memory and learning impairments in rats: Involvement of nuclear factor kappa $B$ and oxidative stress, and prevention by topiramate. J Neurochem. 2010; 114: 675-684

13. Uys JD, Knackstedt L, Hurt P, et al. Cocaine-Induced Adaptations in Cellular Redox Balance Contributes to Enduring Behavioral Plasticity. Neuropsychopharmacology. 2011; 36: 2551-2560.

14. Dietrich JB, Mangeol A, Revel MO, et al. Acute or repeated cocaine administration generates reactive oxygen species and induces antioxidant enzyme activity in dopaminergic rat brain structures. Neuropharmacology. 2005; 48: 965-974.

15. Walker J. Total antioxidant capacity is signi fi cantly lower in cocaine-dependent and methamphetamine-dependent patients relative to normal controls : results from a preliminary study. Hum Psychopharmacol Clin Exp. 2014; 29: 537-543.

16. Bashkatova V, Meunier J, Vanin A, et al. Nitric oxide and oxidative stress in the brain of rats exposed in utero to cocaine. Ann N Y Acad Sci. 2006; 1074: 632-642.

17. Poon HF, Abdullah L, Mullan MA, et al. Cocaine-induced oxidative stress precedes cell death in human neuronal progenitor cells. 
Neurochem Int. 2007; 50: 69-73.

18. Numa R, Kohen R, Poltyrev T,. Tempol diminishes cocaine-induced oxidative damage and attenuates the development and expression of behavioral sensitization. Neuroscience. 2008; 155: 649-658.

19. Numa R, Baron M, Kohen R, et al. Tempol attenuates cocaine-induced death of PC12 cells through decreased oxidative damage. Eur J Pharmacol. 2011; 650: 157-162.

20. Samuni A, Krishna CM, Riesz P, et al. A Novel Metal-free Low Molecular Weight Superoxide Dismutase Mimic. J Biol Chem. 1988; 263: 1792117924.

21. Soule BP, Hyodo F, Matsumoto K, et al. The chemistry and biology of nitroxide compounds. Free Radic Biol Med. 2007; 42: 1632-1650.

22. Cuzzocrea S, McDonald MC, Mazzon E, et al. Effects of tempol, a membrane-permeable radical scavenger, in a gerbil model of brain injury. Brain Res. 2000; 875: 96-106.

23. Thiemermann C. Membrane-permeable radical scavengers (tempol) for shock, ischemia-reperfusion injury, and inflammation. Crit Care Med. 2003; 31: S76-S84.

24. Trembovler V, Beit-Yannai E, Younis F, et al. Antioxidants attenuate acute toxicity of tumor necrosis factor-alpha induced by brain injury in rat. J Interferon Cytokine Res. 1999; 19: 791-5.

25. Liang $Q$ Smith AD, Pan S, et al. Neuroprotective effects of TEMPOL in central and peripheral nervous system models of Parkinson's disease. Biochem Pharmacol. 2005; 70: 1371-1381.

26. Hahn SM, DeLuca AM, Coffin D,. In vivo radioprotection and effects on blood pressure of the stable free radical nitroxides. Int J Radiat Oncol. 1998; 42: 839-842

27. Beit-Yannai E, Zhang R, Trembovler V, et al. Cerebroprotective effect of stable nitroxide radicals in closed head injury in the rat. Brain Res. 1996; 717: 22-28

28. Jang HJ, Hwang S, Cho KY, et al. Taxol induces oxidative neuronal cell death by enhancing the activity of NADPH oxidase in mouse cortical cultures. Neurosci Lett. 2008; 443: 17-22.
29. Youn CK, Kim J, Jo ER, et al. Protective Effect of Tempol against Cisplatin-induced Ototoxicity. Int J Mol Sci. 2016 Nov 18; 17(11): 1-13

30. Ali MR, Abo-Youssef AM, Messiha BA, et al. Tempol and perindopril protect against lipopolysaccharide- induced cognition impairment and amyloidogenesis by modulating brain-derived neurotropic factor, neuroinflammation and oxido-nitrosative stress. Naunyn Schmiedebergs Arch Pharmacol. 2016 Jun; 389(6): 637-656

31. Bernardy CCF, Zarpelon AC, Pinho-Ribeiro FA, et al. Tempol, a Superoxide Dismutase Mimetic Agent, Inhibits Superoxide AnionInduced Inflammatory Pain in Mice. Biomed Res Int. 2017; 2017 :9584819 doi: 10.1155/2017/9584819.

32. Alzoubi KH, Rababa AM, Yacoub ONAl. Physiology \& Behavior Tempol prevents post-traumatic stress disorder induced memory impairment. Physiol Behav. 2018; 184: 189-195.

33. Lee HA, Lee E, Do GY, et al. Histone deacetylase inhibitor MGCD0103 protects the pancreas from streptozotocin-induced oxidative stress and $\beta$-cell death. Biomed Pharmacother. 2019; 109: 921-929.

34. Sadowska-Bartosz I, Bartosz G. Redox nanoparticles: synthesis, properties and perspectives of use for treatment of neurodegenerative diseases. J Nanobiotechnology. 2018; 16: 87.

35. Ben Yehuda Greenwald M, Frušić-Zlotkin M, Soroka Y, et al. Nitroxide delivery system for Nrf2 activation and skin protection. Eur J Pharm Biopharm. 2015; 94: 123-134.

36. Beiser T, Numa R, Kohen R, et al. Chronic treatment with Tempol during acquisition or withdrawal from CPP abolishes the expression of cocaine reward and diminishes oxidative damage. Sci Rep. 2017 Sep 11; 7(1): 11162

37. Jang EY, Ryu YH, Lee BH, et al. Involvement of reactive oxygen species in cocaine-taking behaviors in rats. Addict Biol. 2015; 20: 663-675.

38. Qi C, Wang X, Ge F, et al. mGluR5 in the nucleus accumbens shell regulates morphine-associated contextual memory through reactive oxygen species signaling. Addict Biol. 2015; 20: 927-940.

39. Jang EY, Yang CH, Hedges DM, et al. The role of reactive oxygen species in methamphetamine self-administration and dopamine release in the nucleus accumbens. Addict Biol. 2016; 1-12. 